Revista internacional de fronteras, territorios y regiones / International Journal of Borders, Territories and Regions

FRONTERA NORTE VOL. 33, ART. 15, 2021

https://doi.org/10.33679/rfn.v1i1.2153

\title{
Violación, cuerpo y cognición. Un caso en la Sierra Tarahumara
}

\section{Rape, Body, and Cognition. A Case in the Tarahumara Mountain}

\author{
Rosa Icela Ojeda Martínez ${ }^{1}$
}

\section{RESUMEN}

A través de un estudio de caso, el artículo analiza cómo la práctica de la violación sexual es una forma de presionar a los pueblos originarios para que abandonen sus territorios. Se discute cómo algunos aspectos de la cosmovisión o'dami se relacionan con la cognición corporizada. La metodología de estudio es cualitativa y etnográfica, consistió principalmente en la realización de entrevistas a profundidad. El análisis partió de un enfoque interdisciplinario entre la antropología y las ciencias cognitivas. Los resultados demuestran que la cultura o'dami establece un vínculo entre cuerpo, alma y naturaleza. Se concluye que para analizar la violencia hacia las mujeres y niñas indígenas, es necesario hacerlo de forma integral, incluyendo dimensiones culturales. La limitación principal consistió en la inseguridad pública que impera en la región estudiada, que impidió visitar físicamente la comunidad, por lo tanto, las entrevistas se realizaron en la ciudad de Chihuahua.

Palabras clave: 1. violación, 2. cognición, 3. tepehuanes, 4. Sierra Tarahumara, 5. norte de México.

\begin{abstract}
Through a case study, the article analyzes how the practice of sexual assault is a way to pressure indigenous people to leave their territories. It is discussed how some aspects of the O'dami worldview are related to embodied cognition. The study methodology is qualitative and ethnographic, consisting mainly of in-depth interviews. The analysis departs from an interdisciplinary approach between anthropology and cognitive sciences. The results show that O'dami culture establishes a link between body, soul, and nature. It is concluded that to analyze violence against indigenous women and girls, it is necessary to do so in an integral manner, including cultural dimensions. The main limitation was the insecurity that prevails in the studied region, which prevented visits to the community. Therefore, interviews were conducted in the city of Chihuahua.
\end{abstract}

Keywords: 1. sexual assault, 2. cognition, 3. Tepehuan people, 4. Sierra Tarahumara, 5. northern Mexico.

Fecha de recepción: 24 de agosto de 2020

Fecha de aceptación: 12 de noviembre de 2020

Fecha de publicación web: 15 de agosto de 2021

\footnotetext{
${ }^{1}$ Escuela de Antropología e Historia del Norte de México-Cátedras Conacyt, México, riojeda@conacyt.mx, https://orcid.org/0000-0002-4897-7369
} 
2 Violación, cuerpo y cognición. Un caso en la Sierra Tarahumara

Ojeda Martínez, R. I.

\section{INTRODUCCIÓN}

Atendiendo a la solicitud de la Comisión de Atención a Víctimas del Estado de Chihuahua, realicé un peritaje antropológico para establecer los daños infringidos a una menor de edad del grupo o'dami, o tepehuanos del norte, que fue víctima de violación en la comunidad Cordón de la Cruz, ejido de Baborigame, municipio de Guadalupe y Calvo, en Chihuahua, al norte de México. Este trabajo se desprende de la investigación que se realizó como base argumental de dicho peritaje.

Para entender la violación como un fenómeno psicosocial que afecta no solo a las víctimas directas, sino a todo su grupo étnico, se retomó la hipótesis de Rita Segato (2003, 2014, 2016), que señala que la violación del cuerpo de las niñas y mujeres indígenas en el contexto de la violencia en Latinoamérica, va más allá de la violación por sí misma. Es decir, que esta forma de violencia no persigue únicamente la satisfacción sexual, pues en un plano simbólico está relacionada con el uso del poder de los grupos criminales sobre las comunidades indígenas.

Esta misma propuesta concibe los ataques sexuales en contra de las mujeres como formas de presión usadas contra los grupos étnicos para coaccionarlos y obligarlos a abandonar sus tierras. Bajo esta noción, el cuerpo femenino se revela como campo de batalla en el que los hombres luchan encarnando el triunfo del invasor territorial sobre el indígena (Segato, 2003, 2014, 2016).

La violencia sexual contra niñas indígenas en México es un tema poco abordado por la antropología. La mayoría de las publicaciones sobre violación y otras formas de violencia sexual se centra en mujeres adultas, en zonas urbanas y con población mestiza, a excepción de unos cuantos trabajos, entre los que resaltan Bonfil, Marinis, Rosete y Martínez (2017) y Frías (2014). En las regiones de México donde existen disputas políticas por territorios u otros tipos de conflictos armados, las mujeres y las niñas tienden a ser blanco de la violencia, en parte debido a la falta de mecanismos legales que velen por sus derechos, y a la omisión del Estado (De Marinis, 2013; Sierra, 2008).

Para el caso de la Sierra Tarahumara, existe una escasa documentación sobre el tema, a excepción de Chaparro (2018), y un diagnóstico realizado por Villalobos, Martínez y Carrillo (2018) realizado a través de la Consultoría Técnica Comunitaria A. C. (Contec). En dicho documento se exponen las principales formas de violencia que padecen las comunidades indígenas de esta sierra, incluidas formas de violencia contra las mujeres.

Chihuahua se ha distinguido por sus altos índices de violencia, producto de las prácticas delictivas del narcotráfico. Ciudad Juárez y la Sierra Tarahumara son los principales focos de estas actividades. Ciudad Juárez se sitúa en la frontera entre México y Estados Unidos, esto la convierte en el paso obligado del tránsito de drogas y personas de sur a norte del continente. En la región sur del estado se ubica la Sierra Tarahumara, donde habitan mayoritariamente pueblos originarios. Además, en los límites con los estados de Sinaloa y Durango se encuentra el triángulo dorado, donde se cultiva amapola y marihuana, y se 
produce goma de opio. En esta parte de la sierra, la población ha sufrido las consecuencias de la violencia y el dominio de los grupos criminales (Alarcón y Partido, 2018).

La disposición de este artículo es la siguiente: primero, se analiza la relación entre violencia sexual, cuerpo femenino y territorio; aquí se exponen las principales ideas sobre cómo la violencia sexual en contextos de guerra ha tenido como principal objetivo el cuerpo de niñas y mujeres.

En segundo lugar, se explica la cognición corporizada; además se expone cómo esta propuesta guarda semejanzas con la concepción o'dami sobre el cuerpo. Se remarcan los beneficios de utilizar estas perspectivas para comprender mejor las concepciones del cuerpo o'dami, y cómo este conocimiento puede tener aplicaciones en el marco del acceso a la justicia.

Posteriormente, se explica la metodología que se utilizó en este estudio, y después se expone un apartado sobre algunas concepciones del cuerpo, la salud, la enfermedad y la violación sexual desde la perspectiva de la cultura o'dami. Después se analiza cómo se relaciona el caso de estudio con el despojo de tierras. Finalmente, se ofrece una discusión y conclusiones.

\section{VIOLENCIA, CUERPO FEMENINO Y TERRITORIO}

Toda forma de violencia tiene dos dimensiones: una expresiva y otra instrumental. La primera tiene que ver con la acción, por ejemplo, golpear a alguien para asaltarlo; esto significa que la dimensión expresiva tiene que ver con la violencia misma, con el nivel de daño que se puede causar, con la crueldad. Es decir, con la expresión intrínseca de la violencia. Por otro lado, la dimensión instrumental tiene que ver con los objetivos ulteriores que persigue el agresor (Company, 2014).

En una violación sexual, la dimensión expresiva es confundida con la dimensión instrumental. O sea, sería un error pensar que un violador busca solamente el placer sexual, pues el verdadero fin del coito forzado no consiste en obtener un beneficio inmediato, sino que persigue ejercer poder sobre el cuerpo y la dignidad del otro (Segato, 2014). Es casi una regla que durante conflictos armados, territoriales o interétnicos, la violencia sexual busca expresar humillación hacia el resto de los miembros del grupo familiar y étnico de la víctima (Denov, 2006; Segato, 2014).

La violencia sexual atenta claramente contra la corporeidad de los individuos, lo cual tiene repercusiones inmediatas, y además, violenta la dignidad de las personas. Esta violencia es significada de forma diferente de acuerdo a la cultura. En los conflictos armados, las mujeres de todas las edades son afectadas de forma directa e indirecta, y la violación es una de las formas directas más frecuente. De forma indirecta son afectadas a través del desplazamiento forzado que conlleva la pérdida del hogar, lo que a su vez genera empobrecimiento, separación y desmantelamiento de la familia (Denov, 2006).

Se ha encontrado que en contextos de guerra, la violencia sexual sobre el cuerpo femenino ha seguido un patrón recurrente: los vencedores se apropian del cuerpo de 
4 Violación, cuerpo y cognición. Un caso en la Sierra Tarahumara

Ojeda Martínez, R. I.

mujeres, de niñas e incluso de niños pertenecientes al bando vencido. Aunque niños y hombres adultos también pueden ser violentados sexualmente, la mayoría de las veces los ataques se dirigen contra mujeres y niñas.

Esta violencia presenta variaciones. Por ejemplo, en Bosnia-Herzegovina los serbios sistemáticamente agredieron sexualmente a las mujeres islámicas; en Ruanda, los ataques hacia las mujeres tutsi fueron tan recurrentes que fueron catalogados como genocidio. En cambio, en el conflicto Israel- Palestina, Sri-Lanka y El Salvador, la violencia sexual fue menor. Esto replantea que la violencia sexual contra mujeres y niñas durante los conflictos armados en las distintas regiones del mundo es un fenómeno heterogéneo que no puede ser encasillado en un molde único y universal. Sin embargo, las violaciones en las guerras siempre son una constante (Wood, 2009).

Las formas de violencia sexual más comunes en la guerra son la violación, la esclavitud, la tortura sexual, el matrimonio y el embarazo forzado, la prostitución, las esterilizaciones forzadas y la mutilación genital. Es posible que se obligue a familiares a presenciar las violaciones, y que sean practicadas de forma grupal. El fin que persiguen estos actos consiste en tomar venganza, influir miedo y humillar, sobre todo cuando se escoge a mujeres jóvenes y vírgenes (Wood, 2009).

En Guatemala, el ejército usó las violaciones como una estrategia de terror, utilizándose como forma de castigo y apropiación de las esposas, hijas, madres y hermanas de los guerrilleros. Las violaciones se hacían "con el objeto de paralizar a la población organizada" y para aniquilar toda expresión de oposición, rebelión y resistencia (Fulchirone, Paz, López y Pérez, 2011, pp. 176-177).

En Chiapas, México, desde la década de los noventa se ha vivido una guerra de baja intensidad, en la que la violación en contra de mujeres se ha utilizado para reprimir a los zapatistas. A través de la violación se infringe miedo contra la población, obligándole a huir. La violación en estos contextos se interpreta como una sanción y advertencia en contra de los hombres organizados (Hernández Castillo, 2002).

La violencia sexual contra las mujeres en Latinoamérica ha incrementado a consecuencia de guerras no formalizadas al interior de los países, no solo se presenta en las regiones indígenas, también podemos presenciarla en algunas ciudades importantes, por ejemplo, en San Pedro Sula, Honduras y Rio de Janeiro en Brasil. Las cuales han sido señaladas por diversos organismos internacionales como las zonas más conflictivas del continente. Uno de los aspectos más graves de esta modalidad de guerra radica en la acentuada violencia sexual hacia las mujeres (Segato, 2014).

En México no vivimos una guerra formal, sin embargo, existen formas de violencia que surgen de conflictos entre fuerzas militares, civiles, y narcotraficantes. Este fenómeno conocido como narcoguerra ha sembrado un contexto social de hiperviolencia. La región norte del país representa un necroespacio de violencia constante, conformándola como una práctica naturalizada (Misra, 2018).

En estas regiones dominadas por las narcoguerras localizadas principalmente en países en vías de desarrollo, mujeres, niñas y niños se convierten en los sujetos más vulnerables, 
debido a las estructuras de orden patriarcal, que confiere a los hombres la concentración del poder físico, político y económico. En estos conflictos las prácticas violentas sobre el cuerpo como objeto de ultraje, lucha territorial y colonización, muestran que a menudo la corporalidad femenina puede adoptar significados asociados a la idea de superficie, cartografía y tenencia territorial, un locus físico y simbólico sobre el cual se trazan las normas de la crueldad (Acosta, 2018; Milán, 2017; Segato, 2014).

\section{LA IMBRICACIÓN CUERPO-MENTE Y LA COGNICIÓN CORPORIZADA}

La explicación occidental de qué es y cómo funciona la mente humana ha provenido tradicionalmente de las ciencias cognitivas, sobre todo de la psicología, la inteligencia artificial, la filosofía. Aunque la antropología cognitiva ha realizado investigaciones importantes, su rol ha sido más marginal. Lo anterior tal vez obedece a que la antropología se ha centrado en estudiar modelos de la cognición humana de culturas no occidentales (D’Andrade, 1995).

En un principio el modelo occidental de la mente, creado por las ciencias cognitivas, estuvo dominado por la idea de que la mente humana funciona de forma muy parecida a una computadora, es decir, como un procesador de símbolos. Esta concepción sobre la naturaleza de la mente implicó que la actividad mental se circunscribiera exclusivamente al cerebro, y que el resto del cuerpo quedara desligado de todo proceso cognitivo. De la misma manera, el contexto cultural y el medio ambiente fueron relegados a un papel secundario.

Este modelo postulaba que toda inteligencia descansa en la capacidad de representar el mundo, a través de una actividad subjetiva e individualizada. Esta visión internalista de la cognición tiene como hipótesis central que la mente humana funciona a través de representaciones simbólicas que sólo ocurren dentro del cerebro del agente, es decir, que todo sistema inteligente funciona como un procesador de símbolos independiente del medio ambiente (Varela, Thompson y Rosch, 2011).

Bajo esta perspectiva la mente está descorporizada y lo que ocurre dentro de ella se desliga del resto del mundo. Es decir, hay una diferencia muy marcada entre el mundo interno, subjetivo y el mundo externo, que estaría totalmente ajeno y desconectado del mundo mental del sujeto. "en otras palabras, la principal característica de la mente, según el cognitivismo, era su aislacionismo ontológico, o lo que es lo mismo, que la mente se encuentra en un espacio compuesto de estados internos independientes del cuerpo" (Vélez, 2009).

Por el contrario, en el enactivismo y la cognición corporizada se pone énfasis en la relación cognición-cuerpo-mundo, así la experiencia humana recae sobre el hecho de que encarnamos un cuerpo-mente que vive un mundo social, cultural y material determinado (Lindblom y Ziemke, 2008). 
6 Violación, cuerpo y cognición. Un caso en la Sierra Tarahumara

Ojeda Martínez, R. I.

Retomando la perspectiva de la cognición corporizada, la psiquiatría cultural ha puesto de relieve cómo la variación cultural moldea la experiencia corporal sobre síntomas asociados a enfermedades psiquiátricas, poniendo en entredicho la supuesta universalidad de algunos síntomas, y resaltando que los individuos interpretan la enfermedad de acuerdo a sus marcos culturales (Kirmayer y Ramsted, 2017). Esto demuestra que es trascendental incluir la cultura, sobre todo la cosmovisión de los pueblos, a fin de entender cómo eventos traumáticos en las vidas de las personas pueden generar distintos daños, esto si tomamos en cuenta que un modelo de la mente humana no es universal, sino que está fuertemente enlazado a las particularidades del contexto social y cultural.

La cognición corporizada retoma algunas propuestas de la antropología del embodiment, por ejemplo, los trabajos de Csordas (1990) y de Durt, Fuchs y Tewes (2017). Esto muestra que la interacción entre las ciencias cognitivas y la antropología puede ser fructífera para analizar modelos no occidentales de la cognición.

En México se ha comenzado a tomar con seriedad la posibilidad de la cognición 4E (por sus siglas en inglés) corporizada, empotrada, extendida y enactiva (Gonzalez-Grandón y Froese, 2018). Una alternativa particularmente radical ha sido desarrollada por la aproximación enactiva, que rechaza el paradigma tradicional internalista-individualista de la mente. Dicho paradigma ha sido perseguido por el escenario del "cerebro en una cubeta", donde los factores externos del cerebro son reducidos solamente a posibles patrones de entrada o inputs, al contrario de un paradigma que concibe la mente humana como un estar en el mundo corporal, social y culturalmente constituido (Froese, 2019).

Este cambio de paradigma ha desarrollado debates filosóficos que pueden parecer bastante apartados de las preocupaciones concernientes al mundo de la vida concreta, pues generalmente la filosofía que da base al paradigma enactivista se ha aplicado más al desarrollo de aspectos teóricos, o relacionados con la inteligencia artificial. Sin embargo, se argumenta que la elección del paradigma de la mente tiene un impacto en la vida real (Froese, 2019), lo cual se ilustra muy bien en las posibles consecuencias del presente caso de violación por parte de dos hombres supuestamente asociados a un grupo de narcotraficantes. Lo que está en juego aquí es poder delimitar la magnitud del daño ocasionado por este crimen en una escala individual y cultural.

La visión del mundo indígena interpretada desde el punto de vista de Segato (2003, 2014, 2016) concebiría este evento no solo como una violación hacia una niña, sino también a su familia, a su comunidad, a su grupo étnico entero, e incluso a la tierra que habitan. Por otro lado, la perspectiva o'dami sobre el cuerpo entrelaza en un continuum cuerpo-alma-medioambiente, lo que no encaja fácilmente con un paradigma internalista individualista, el cual implicaría que el daño y sus posibles reparaciones se concentrarían en el daño provocado exclusivamente a la niña, principalmente el ocasionado al cuerpo físico y psíquico. Pero el daño a nivel de la familia, la comunidad, e incluso al grupo 
cultural completo, sería tratado como algo externo, y por lo tanto, como sujeto a consecuencias separadas.

La aproximación enactiva, por otro lado, concuerda con la perspectiva indígena de que la mente y el cuerpo no pueden ser separados, e incluso va más lejos, al aceptar que el cerebro, el cuerpo y el medio social forman un todo significativo (Fuchs, 2018), lo cual tiene implicaciones notables en la concepción de la salud y sus desórdenes (Kirmayer y Ramsted, 2017). El daño infligido debe, por lo tanto, ser evaluado de una manera culturalmente sensible. De esta forma, los avances recientes de la filosofía y las ciencias de la mente permiten apreciar mejor el daño infligido a las poblaciones indígenas a través de esta forma de violación dirigida, y permite hacerlo de una manera que es más consistente con su propia cosmovisión holística.

La aproximación corporizada deja entender de forma más adecuada la relación entre cuerpo y mundo natural que subyace a la cultura o'dami, donde el cuerpo, la salud y la enfermedad no se reflejan únicamente en el cuerpo físico, sino que va más allá y se entrelaza con el medio ambiente, la naturaleza y el plano espiritual. Esta concepción confirma que los modelos de la psique propuestos por el cognitivismo clásico, que por mucho tiempo han representado el modelo hegemónico de la cognición, no reflejan la variabilidad cultural y, desde mi punto de vista, lo más importante es que la cultura o'dami puede enseñarnos una visión alternativa sobre el entrelazamiento cuerpo-cognición y mundo.

\section{METODOLOGÍA}

La metodología comprende un acercamiento etnográfico fundamentado principalmente en la realización de entrevistas a profundidad, por lo tanto la naturaleza de los datos y su análisis es totalmente cualitativa. La perspectiva de estudio incluye un diálogo entre la antropología y las ciencias cognitivas.

Se realizaron 11 entrevistas a la familia de la víctima y ocho a otros miembros del pueblo o'dami que han migrado a la ciudad de Chihuahua y al municipio de Guachochi, en el estado de Chihahua. El trabajo de campo se realizó en tres etapas; la primera se llevó a cabo de abril a mayo de 2018, la segunda etapa comprendió de noviembre a diciembre de 2019, y una última, de mayo a septiembre de 2020. De forma paralela, se realizó una investigación documental sobre la cultura o'dami, se revisó la carpeta de investigación judicial de la denuncia interpuesta por parte de la familia a las autoridades, que incluía la narración de los hechos el día del ataque, el peritaje médico y el peritaje psicológico. Por cuestiones de seguridad, fue imposible visitar personalmente la comunidad donde ocurrieron los hechos, y por cuestiones éticas y recomendaciones de especialistas en psicología, la víctima directa no fue entrevistada. 
8 Violación, cuerpo y cognición. Un caso en la Sierra Tarahumara

Ojeda Martínez, R. I.

Las entrevistas se audio grabaron y después se transcribieron usando el software Transcriber 1.5.1; posteriormente, las entrevistas se analizaron de forma cualitativa. Es decir, se hizo un análisis interpretativo profundo de las narraciones de los informantes tal como las enuncian, tratando de entender el significado antropológico de las situaciones sociales e intersubjetivas que ellos exponen (Salgado, 2007). También se corroboraron algunos datos etnográficos con especialistas que han realizado trabajo de campo en la región. Para la realización de la investigación se siguió un enfoque ético, basado en los principios del proyecto ERIC: Ethical Research Involving Children (Granham, Powell, Tylor, Anderson y Fitzgerald, 2013). En el presente texto se usarán seudónimos para nombrar a la víctima y sus familiares.

\section{EL ATAQUE}

Los padres de Esperanza no estaban en casa, su mamá se había ido con sus tíos al pueblo de Baborigame a celebrar un tónare, una especie de festejo ritual donde se come carne y se toma tesgüino, una bebida fermentada de maíz que se consume en fiestas y rituales. El papá, a quien nombraré como Benito, había salido acompañado de otros tepehuanes a interponer una demanda por el despojo de tierras ejidales. Los quejosos ya habían intentado demandar en el pueblo de Baborigame, pero no les hicieron caso, así que fueron al municipio de Guadalupe y Calvo y tampoco los escucharon, por eso decidieron ir a la capital del estado, Chihuahua, y allá andaban cuando ocurrieron los hechos.

En la casa se habían quedado sólo los menores, Leticia, de 16 años, Efraín, de 12, Esperanza, Jacinto y Roberto, de 10, Andrés, de 8 y Jorge, de 7 años. Para los pequeños parecía un día cualquiera, regresaban de cortar leña acompañados de sus perros cuando de pronto se escuchó que se acercaba una camioneta, todos corrieron a la casa a ver quién era.

Del vehículo salieron tres hombres; conocían bien a dos de ellos, al otro no lo reconocieron porque traía cubierto el rostro con un pasamontaña, como el que usan los soldados. El que daba las órdenes era Carlos, lo reconocieron, pues era sobrino del hombre que se había apropiado de las tierras de su familia.

Días antes Carlos había amenazado a Benito. En aquella ocasión el primero había ido a reclamar por qué andaba haciendo tanto alboroto por lo de las tierras, pero como no lo encontró, mató a balazos a un perro que estaba en la entrada de la casa y amenazó a la familia. Esta vez Carlos también venía dispuesto a hacer daño; se metió a la casa y comenzó a romper los pocos muebles que había adentro, a cambio de las tierras en disputa pedía a los menores dinero a gritos, decía que los iba a matar.

Leticia salió corriendo para el monte hasta llegar a una de las casas vecinas, el resto intentó huir, pero no lo lograron. La pequeña Esperanza quedó atrapada dentro de la casa, lo único que pudo hacer fue meterse debajo de la cama. Los hombres alcanzaron a agarrar a Efraín, quien intentaba huir, lo comenzaron a golpear de forma salvaje, le dieron patadas 
en la cara, la cabeza y en todo su pequeño cuerpo, después lo amarraron de las manos y lo ataron a un árbol. A Jacinto lo arrastraron, lo metieron a la casa y le ordenaron que observara.

Carlos sacó a la fuerza a Esperanza de abajo de la cama, la agarró del cuello y la apretó muy fuerte. La niña apenas podía respirar, la aventó con fuerza sobre la cama, le dijo que si ponía resistencia mataría a Jacinto, y que después la mataría a ella. Carlos sacó una navaja, Esperanza no dudó en luchar e intentó quitársela, pero sólo logró cortarse la mano, ella luchaba, pero el hombre era muy fuerte, sus esfuerzos eran en vano.

Esperanza gritaba pidiendo ayuda, entonces Carlos ordenó al segundo hombre que violara a la niña, que lo hiciera rápido. Carlos se acercó decididamente a Jacinto y lo tomó de la cara, obligándolo a observar la brutal escena, Jacinto no quería ver, tenía mucho miedo y se resistía, mientras su atacante lo amenazaba y le repetía que si le contaba a alguien, lo mataría. Después Carlos violó a Esperanza con la misma crueldad que su cómplice. Jacinto aprovechó el descuido de los hombres y salió corriendo lo más rápido que pudo, alcanzó a llegar a un sembradío de maíz que estaba cerca de la casa y ahí se quedó inmóvil.

El tiempo para Esperanza fue eterno, nunca dejó de gritar y de pedir ayuda; sus gritos quedaron resonando en las paredes de aquella casa, que hoy está abandonada. Finalmente, los hombres se fueron, Esperanza salió corriendo y se escondió entre el sembradío de maíz, donde pasó toda la noche temiendo que los hombres regresaran. Al día siguiente encontró a Efraín y a Jacinto, ellos también habían dormido a la intemperie, escondidos entre la siembra; Efraín todavía sangraba y tenía atadas las muñecas.

Cuando la mamá regresó, supo que Carlos había ido y que había hecho destrozos en la casa, vio a su hija golpeada y sin ánimos, nada más acostada, no quería comer y se quejaba de dolor de estómago, pero la niña no le contó nada de lo sucedido porque tenía miedo. De la violación se enteró después, cuando los otros niños le dijeron lo que había pasado. Rápidamente la llevó al hospital de Baborigame, pero ahí no la quisieron atender. La mamá contó que Carlos tenía conocidos en el hospital, estos se negaron a atender a la niña. Entonces se fue hasta la cabecera municipal y ahí sí la atendieron.

El reporte del médico confirmó la violación, además, el ataque había tenido consecuencias en su cuerpo, resultado de la lucha que sostuvo con los agresores. El informe psicológico también confirmó la presencia de un agudo estrés postraumático, el cual se prolongaría por tiempo indefinido si no recibía atención psicológica.

El proceso de los peritajes psicológicos y la demanda fue revictimizante, pues la niña sentía vergüenza de narrar una y otra vez lo que había ocurrido. no podía hacerlo en español, por lo que además del psicólogo, médicos y funcionaros, requirió de un traductor, convirtiendo el proceso de entablar la denuncia en un acto público que generó angustia y vergüenza en la niña, así lo constató el reporte psicológico. 
10 Violación, cuerpo y cognición. Un caso en la Sierra Tarahumara

Ojeda Martínez, R. I.

En un primer momento, la denuncia se interpuso en el municipio de Guadalupe y Calvo, pero no se le daba celeridad al proceso, ya que la familia de Carlos tenía relación con las autoridades Ministeriales. Con ayuda de una organización civil, la familia de Esperanza logró que la Comisión Estatal para los Pueblos Indígenas (COEPI) interviniera para que el caso se llevara a la fiscalía especializada en atención a mujeres víctimas del delito por razones de género, en la ciudad de Chihuahua. Debido a todo esto, amenazaron de muerte al padre de Esperanza, y según él, incluso ofrecían una suma de dinero por su cabeza, así que la familia tuvo que irse a vivir a la capital.

Los padres refirieron que después de la violación, sus vidas cambiaron completamente, sobre todo el hecho de tener que mudarse a vivir a la ciudad, pues desde ahí no pueden frecuentar a sus familiares y amistades. Al abandonar sus tierras de trabajo, dejaron de percibir ingresos, su ganado murió, su casa fue abandonada y destruida junto con su huerto y sus "animales de patio". El papá siempre se había dedicado al campo, y además tenía un trabajo extra como auxiliar de salud, pero al abandonar la ranchería ya no pudo desempeñar ninguna de sus labores y por un buen tiempo estuvo desempleado, sin forma de obtener ingresos.

La violación afectó el prestigio social de la familia, pues los padres de Esperanza mencionaron que en la escuela los niños se burlaban de que ella había sido violada, al grado que ella que tuvo que abandonar la escuela. Al parecer, el conflicto por las tierras, los antiguos ataques que había recibido la familia y la violación de la niña, provocaron que algunos miembros de su comunidad les dejaran de hablar.

\section{LA VIOLACIÓN EN EL CONTEXTO DEL DESPOJO DEL TERRITORIO O'DAMI}

Los conflictos territoriales en la Sierra Tarahumara se remontan a la época de la colonia, cuándo los españoles abrieron las primeras minas y misiones en territorios indígenas. Los colonizadores establecieron campamentos para explotar las minas, y fundaron misiones evangelizadoras en las tierras que no les pertenecían; estos son los primeros despojos que sufrieron los pueblos originarios que habitan la Sierra Tarahumara.

En la actualidad estos pueblos siguen luchando para defender sus territorios ancestrales, los o'dami han sido aguerridos, es muy conocida su oposición y resistencia a la aculturación desde la llegada de las primeras misiones y conquistadores españoles (De la Cruz, 2008). Hasta el día de hoy, los o'dami continúan siendo victimas de despojos por parte de familias de caciques mestizos que se atribuyen la propiedad de sus tierras.

En los años sesenta, el gobierno entregó 3500 hectáreas en el municipio de Guadalupe y Calvo a una sola propietaria mestiza, pasando por alto que estas tierras estaban ocupadas por el pueblo o'dami. En 1981 los tepehuanes reclamaron legalmente sus tierras, pero se han enfrentado a la lentitud de los tribunales y a la presión por parte de los mestizos, que se adjudican la propiedad. Recientemente exigieron a las autoridades agrarias que se les 
reconociera la propiedad de esas tierras y que se respetaran sus derechos colectivos como pueblos originarios (Breach Velducea, 2007).

En el año 2019 las autoridades tradicionales denunciaron un nuevo despojo de tierras por parte de una familia mestiza en la ranchería Cordón de la Cruz; esta familia había hecho una solicitud a la Secretaría de Desarrollo Agrario Territorial y Urbano para ocupar territorios que han sido habitados por los o'dami desde tiempos ancestrales. Esta familia, creyéndose propietaria legal, ha hostigado a las familias que viven en estos ranchos (Raíchali Noticias, 2019). Este ultimo caso es el que tiene consecuencias directas para la presente investigación, pues uno de los miembros de esta familia fue el que abusó sexualmente de Esperanza.

La ranchería Cordón de la Cruz, lugar donde ocurrieron los hechos, pertenece a la sección de Baborigame, en el municipio de Guadalupe y Calvo; este municipio ha sido clasificado como uno de los más violentos de todo el país. La última década ha sido particularmente violenta en la Sierra, este clima ha orillado a las familias a huir a la capital del estado y a otros centros urbanos cercanos para protegerse de las amenazas de muerte por parte de narcotraficantes (Breach Valducea, 2016).

Para entender la violación en contra de Esperanza es necesario ubicarse en este contexto, ya que no ocurrió como un hecho aislado, sino que sucedió en el marco de una serie de ataques que ha sufrido su familia y su comunidad.

Según refiere la familia de Esperanza, hace algunos años el abuelo paterno fue asesinado por conflictos territoriales; también la abuela materna y su esposo fueron encontrados descuartizados en su casa, sin que hasta la fecha se haya encontrado a los culpables. La violación a Esperanza no es un acto aislado, sino que forma parte de las prácticas que los narcotraficantes han emprendido constantemente en contra de las comunidades indígenas para presionarlos a abandonar sus hogares y apropiarse ilegalmente de sus territorios.

Es necesario entender que este despojo en la Sierra Tarahumara no es un evento aislado, sino que forma parte de todo un contexto social complejo. Por ejemplo, en las últimas tres décadas el despojo de tierras, que posteriormente han sido usadas para la siembra de marihuana y de amapola, ha convertido a la sierra en un sitio de peligro latente. Esto ha desatado múltiples formas de agravio contra la vida de los pueblos indígenas, principalmente a través de la extracción ilegal de madera de sus bosques, la expropiación de sus territorios, la expulsión de familias enteras de sus comunidades, el robo de ganado, las torturas, los homicidios, la quema de casas, de masacres, y el reclutamiento forzado de jóvenes, así como el desplazamiento forzado (Villalobos, Martínez Esparza y Carrillo Domínguez, 2018).

Todos estos factores convierten el fenómeno de la violencia en la sierra chihuahuense en algo que debe de ser entendido de forma compleja; es decir, las violencias que se viven en esta región no pueden ser atribuidas a una sola causa. Además, este ambiente genera impunidad e impacta negativamente en la vida de los pueblos originarios, de manera 
12 Violación, cuerpo y cognición. Un caso en la Sierra Tarahumara

Ojeda Martínez, R. I.

particular a las mujeres y a las niñas, quienes generalmente son objeto de la violencia sexual.

\section{LAS NOCIONES DE CUERPO, SALUD, ENFERMEDAD Y VIOLACIÓN ENTRE LOS O'DAMI}

Para los o'dami, la concepción del ciclo de la vida es la base de su representación de la naturaleza humana, pues el ser humano se desarrolla a través de diferentes etapas, las cuales no solo implican un desarrollo físico, también abarcan un desarrollo social y espiritual, por así decirlo, pues el concepto de persona incluye el dominio corporal y las almas (Rosas Mérida, 2006, 2012). Dentro de su cosmovisión, los hombres poseen tres almas y las mujeres cuatro, porque son las dadoras de vida (Saucedo Sánchez de Tagle, 2004).

Su universo se divide en tres regiones: el cielo, la tierra y el mundo de abajo. El cielo es habitado por Dios y su esposa ("el que es padre y la que es madre"), entidades asociadas con el sol y la luna; junto a ellos viven sus hijos (que fungen como ayudantes en su interacción con los hombres), y las almas de aquellos que en vida fueron consecuentes con las enseñanzas éticas y morales otorgadas por Dios. Así, toda conducta y todo pensamiento concebidos como socialmente apropiados se relacionan con Dios. En este sentido, reconocerse como indígena representa una liga indisoluble con los principios y los seres asociados con el nivel celeste (Saucedo Sánchez de Tagle, 2004, p. 27).

En contraste, el mundo de los mestizos representa la esfera de lo inmoral y de las malas conductas. Esto significa que los no indígenas y mestizos provienen de naturalezas distintas, pues los primeros son hijos de Dios, y los segundos, del diablo, tal y como se expone en la siguiente cita:

En el otro extremo del cosmos, en el mundo de "abajo", vive el hermano menor de Dios, el diablo, padre de los obhai o chabochis (es decir, los blancos), quien junto con las almas no indias y algunos seres y animales que lo socorren, aconsejan a los indígenas pelear, robar, matar, hechizar, cometer adulterio o cualquier otro tipo de práctica contraria al comportamiento socialmente establecido (Saucedo Sánchez de Tagle, 2004, p. 25).

Cuando le pregunté a Eulogio, un antiguo representante y autoridad indígena tepehuano, sobre la división del universo en tres regiones, contestó lo siguiente:

Aquí está la división. Aquí en este planeta que estamos aquí... aquí habemos personas malas, ahí no dice que vamos pa' el infierno, es al infierno pa' abajo. $\mathrm{Pa}^{\prime}$ la gloria es pa' arriba. Los seres humanos buenos, que no deben o no temen a nada, o no deben a nadie, aquí no deben ni una. Entonces esos son los que van a la Gloria [...] Aquí en el centro está por eso está la división, el malo de aquí va pa' abajo y el bueno pa' arriba. Bueno, esa es la costumbre de, ¿de muy antes?, [de] lo que se ha formado el mundo, así, la creencia (Eulogio Hernández, comunicación personal, 29 de septiembre de 2020). 
Podemos notar cómo las acciones en este mundo van a determinar a dónde va el alma después de la muerte. Para los o'dami es muy importante el comportamiento moralmente aceptado; como veremos más adelante, las violaciones sexuales son vistas como un mal comportamiento, no solo por parte del agresor, sino que la víctima también paga una sanción social y espiritual. La salud es muy importante para los o'dami, pues ésta significa:

[...] tener fuerzas para vivir y trabajar. Asimismo, la idea de salud se relaciona con el hecho de tener dentro del cuerpo todas las almas. La enfermedad, la disminución de la fuerza, sobreviene cuando una persona ha perdido una de sus almas. [...] Si las almas abandonan el cuerpo de manera definitiva, el individuo muere. Cuando alguien se enferma, es común que se solicite los servicios de un médico tradicional, el matikami, quien no sólo atiende al enfermo, sino a toda la familia (Saucedo Sánchez de Tagle, 2004, p. 27).

Las concepciones de salud/enfermedad rebasan el plano físico y corporal, abarcan lo humano y lo no humano; el entorno y la naturaleza conforman un todo en equilibrio con el sujeto. Cuando este equilibrio se rompe, viene la enfermedad y la salud física y espiritual se afectan; la salud depende del buen cuidado del cuerpo y de las almas, de la relación de éstos con la naturaleza y el entorno, como bien explica Martha en el siguiente fragmento:

Cuando yo era niña mi abuela me contó que las personas que cortan los árboles o que dañan la naturaleza se enferman de enfermedades incurables y se mueren. Hay unos árboles que se dan donde hay agua; si las personas los cortan, el ojo de agua se seca y esas personas se enferman (Martha Hernández, comunicación personal, 7 de noviembre de 2019).

La noción de persona se encuentra ligada a la del cuerpo, el alma y la naturaleza, y aunque no existe en su lengua una palabra específica para designar el concepto de naturaleza, los animales y otros elementos del mundo que forman parte de ella son catalogados como seres con alma; de esta forma, existe una continuidad ontológica entre los humanos y otras formas vivas (Reyes et al., 2015). Para los o'dami los elementos de la naturaleza forman parte del mundo humano y afectan su salud física y psíquica, esto refleja la interconexión entre el mundo circundante y el mundo psíquico de las personas.

En los aguajes hay árboles que no deben cortarse porque se dice que el agua se seca, la persona se enferma, muchos así lo hacen. Una vez fui a darle agua a un macho, a un aguaje que era bien bravo; el agua le hacía asina (mueve su mano en círculos), el aguaje gritaba, ese aguaje se comía a las vacas. El aguaje se enoja si la gente tira basura o corta los árboles, a mí me daba miedo ir, también se enojan con uno, no les gusta que los hagan enojar. Esa vez, cuando me asustó, yo nada más llenaba los galones y me arrendaba para la casa rápido para que no me asustara; a veces los aguajes se enojan en la noche, como que chiflan (Ernestina Clemente, comunicación personal, 7 de junio de 2020).

Lo que cuentan Martha y Ernestina testifica que el comportamiento del individuo que afecta a la naturaleza puede a la vez afectar su cuerpo y su alma, incluso ocasionar la muerte. Esta forma de concebir al ser humano y su relación con el mundo, revela que 
14 Violación, cuerpo y cognición. Un caso en la Sierra Tarahumara

Ojeda Martínez, R. I.

cuerpo y mundo se conforman en unidad indisociable. Esta perspectiva o'dami sobre el universo humano y lo que le rodea guarda grandes semejanzas con la perspectiva de la cognición corporizada ya mencionada.

Por otro lado, preguntarse cómo concibe la cultura tepehuana la violación sexual es un tema que resulta muy complicado de abordar, fue clara la sensación de que a las personas no les gusta conversar sobre el tema, al parecer resulta un asunto vergonzoso, el cual se puede eludir fácilmente. Después de más de un año y varios encuentros, pude hablar abiertamente sobre ello con mis informantes. Así, Catalina describe las repercusiones que sufre una mujer o'dami al ser violada por un chabochi (mestizo):

Pues es que ahí es como cuando dicen, este hombre, si fue brutalmente violada, entonces la mujer dice, pues este ya manchó mi alma, y que ya no se siente bien ante los demás, no, y como y aun peor si fue un chabochi, si la violó un chabochi peor siente manchada de su alma; siente que ya no va a ir con Dios, siente que se va a ir con el diablo, porque fue ensuciada por un chabochi (Catalina Hernández, comunicación personal, 29 de septiembre de 2020).

Como podemos ver, el daño más grave no es concebido como un daño físico, ni social, sino como un daño todavía mayor, como daño espiritual. Son la mujer y su alma las que pagan las consecuencias, la imposibilidad de entrar al mundo de Dios, y su sentencia para "ir" con el diablo, asociado a la vida inmoral que lleva el mestizo.

Para entender cómo perjudica la violación sexual en un sentido amplio, es necesario comprender que los daños no solo se infringen a nivel físico y psicológico. También tendríamos que hablar de un daño a nivel socio-cultural, pues para los o'dami las violaciones sexuales son llevadas a cabo sobre todo por los mestizos, implican daños culturalmente situados, pues desde el punto de vista de su cosmovisión también el alma de la víctima se ve seriamente afectada.

Desde esta perspectiva, el cuerpo violado se considera enfermo, en primer lugar y por causas obvias, debido a las heridas y a los golpes, los cuales pueden ser notorios a simple vista, y los daños físicos en órganos internos pueden ser corroborados por un médico. En este caso los daños físicos eran visibles y fueron corroborados por un médico. El daño más severo se proporciona al nivel de las almas, el cuerpo de la mujer que ha sido violada repercute en lo que podríamos llamar la salud de las almas, ya que el alma queda manchada ante los ojos de Dios, y ante los ojos de los demás. Y si el que cometió el abuso es un chabochi o mestizo, el daño es mayor, pues los mestizos son asociados al mundo de abajo, el mundo del diablo. Cuando más de una vez preguntamos si era posible que una mujer o un hombre o'dami se casara con un hombre o mujer chabochi, la respuesta siempre fue afirmativa, ya que de hecho sucedía, pero que, en ese caso, la mujer o el hombre o'dami no iría al cielo cuando muriera.

En la cultura tepehuana las mujeres son las dadoras de vida. Es importante que una mujer llegue virgen al matrimonio, por lo que las mujeres que se sospecha que han estado 
con más de un hombre, carecen de prestigio y son relegadas (Balbuena, 2007). Para ellos, el comportamiento moral es trascendental en este mundo y después de la muerte. La existencia depende del equilibrio entre sentir y pensar, de lo contrario se desencadenan consecuencias como la enfermedad, la muerte y el sufrimiento tanto en el plano terrenal como después de la muerte (Reyes Valdez, Oseguera Montiel, Pacheco Bribesca y Saucedo Sánchez de Tagle, 2015).

A partir de las concepciones o'dami sobre la persona y el cuerpo humano que señalan Balbuena (2007) y Rosas Mérida (2006), se puede inferir que, para los tepehuanes del norte, ser víctima de violación cuando eres niña podría entenderse como una especie de anomalía social del desarrollo de la persona. Como las niñas y los niños no son considerados personas totalmente acabadas, convertirse en persona implica pasar por una serie de etapas de desarrollo consecutivas, que no pueden saltarse. De esta manera, si una niña es violada pierde su virginidad antes de tiempo, pues las relaciones sexuales normalmente ocurren hasta llegar al matrimonio, y las mujeres que tienen relaciones sexuales antes de éste rompen el equilibro individual, social y colectivo (Rosas Mérida, 2006).

Por otro lado, generalmente la violencia sexual no es denunciada por las mujeres tepehuanas, debido al miedo a las represalias, o por miedo a que las personas las "tachen de mujer fácil, prostituta y demás", o porque muchas de ellas no hablan español (Chaparro, 2018, p. 123). Cuando una violación sexual ocurre y es denunciada a las autoridades tradicionales, generalmente se remite a las autoridades mestizas, pues se les considera un delito grave. Sobre este asunto le pregunté a Eulogio Hernández, quien ha sido auxiliar de las autoridades indígenas:

Ah no, eso sí. Ese sí lo pasamos a la autoridad civil competente, eso sí; eso lo tengo que pasar yo [...] No nos las podemos arreglar nosotros porque sería delito federal ya, muy grande ya, eso ya no. [...]. No, pos' a esas, ahí se encarga la autoridad acá, si los casan o como los separan también, o como digan ahí, pero allá tienen que pagar ya (Eulogio Hernández, comunicación personal, 29 de septiembre de 2020).

En el fragmento anterior es claro cómo las violaciones son consideradas un delito grave que amerita ser denunciado a las autoridades mestizas. Pero, al igual que en muchos pueblos originarios de México, una solución puede ser que la víctima se case con su agresor, como una forma de restauración del daño. Esta forma de justicia visiblemente atenta contra los derechos de las mujeres, como dicen Lang y Barrera Vivero (2009):

[...] en los casos que afectan los derechos específicos de las mujeres, por ejemplo, casos de violencia de género, la justica ancestral en la mayoría de las culturas indígenas de América, al igual que la justicia ordinaria, no responde a las condiciones y situaciones especificas de las mujeres indígenas, dejando en desventaja a aquellas mujeres que buscan protección ante ellas $-\mathrm{y}$ en muchas ocasiones, violando sus derechos (Lang y Barrera Vivero, 2009, p. 10). 
16 Violación, cuerpo y cognición. Un caso en la Sierra Tarahumara

Ojeda Martínez, R. I.

\section{DISCUSIÓN}

Cuando una niña o un niño es víctima de un acto de violencia sexual, pueden observarse daños físicos a nivel corporal, pero también se desencadenan daños emocionales y en la salud mental, como ansiedad y depresión. Por otro lado, se presentan perjuicios de orden psicosocial, como son el desarrollo de una baja capacidad para relacionarse con los demás, falta de empatía o aislamiento. Otra constante es que la niña o el niño que ha sido violentado sexualmente puede experimentar el rechazo o la devaluación social (Ramos, 2009).

Las complicaciones a nivel corporal van más allá de la dimensión somática. Especialmente en este caso, bajo la cosmovisión o'dami el cuerpo de Esperanza fue afectado también en el plano espiritual, a nivel de su alma. Además, el cuerpo no se circunscribe únicamente a las fronteras de la piel y el individuo, sino que tiende hilos e interconexiones hacia otros elementos del mundo. Esto tiene resonancia en el marco de la cognición corporizada y enactiva, la cual propone que existe un continuo entre subjetividad, cuerpo y mundo. Esta perspectiva ofrece una base para poder argüir el daño infringido sobre la niña como un daño cultural.

Desde el paradigma internalista-individualista se asume que el estado psicológico de un individuo puede ser totalmente independiente del contexto relacional que lo rodea (Videla y Torrejón, 2019). Esta perspectiva se separa de la propuesta enactiva de la cognición, donde la cultura, el contexto social y el ambiente conforman un todo con la mente; el enactivismo ofrece una visión holista de la relación cuerpo-mente-mundo, en completa concordancia con lo observado en la cultura o'dami. Para entender la violencia contra Esperanza, no es apta la perspectiva individualista, sobre todo cuando observamos cómo el daño infligido a la niña va más allá de su propio cuerpo, en tanto materialidad biológica.

Para los tepehuanes, el cuerpo humano no representa únicamente procesos fisiológicos, el cuerpo otorga asiento a la persona, pero ésta es concebida desde un plano social, colectivo y simbólico. Así, por medio del cuerpo, el ser humano está en comunicación con los diferentes campos simbólicos que le otorgan sentido a la existencia colectiva (Rosas Mérida, 2006).

Según las entrevistas que se realizaron a los padres de Esperanza, su comunidad no se mostró empática con la niña después de la violación. Al contrario, la niña fue revictimizada mediante chismes y burlas. Podemos interpretar esto como una forma de categorizar a Esperanza y su familia como culpables de lo sucedido, por denunciar el despojo de tierras. También podemos interpretar este comportamiento de rechazo aludiendo a lo que Montes y Salice (2017) refieren como un fenómeno de orden psicosocial relacionado con la necesidad de alejarse de las personas o de las situaciones en las que nos sentimos avergonzados, lo que podríamos llamar una especie de vergüenza colectiva, como si nos pudiéramos contaminar socialmente de las vergüenzas y situaciones bochornosas de los otros con los que me identifico. 
La violación podría ser entendida como una especie de ruptura de la armonía colectiva: que Esperanza, al haber sido ultrajada sexualmente, no representa a una persona sana. Como mencionamos páginas atrás, según la cosmovisión o'dami una persona se constituye por un cuerpo y cuatro almas en equilibrio, por lo tanto, la niña pudiera representar un caso típico de la perturbación que acarrea la enfermedad del cuerpo y las almas, un cuerpo violado y contaminado a causa de la maldad natural de los obhai, o mestizos.

Es notorio que los daños que provocan la violencia sexual hacia las mujeres y las niñas no sólo afectan a nivel individual, sino que los perjuicios van desde el orden familiar hasta el colectivo. Se reconocen tres tipos de victimización. La primaria, que es el daño directo. La secundaria, que se refiere a los sufrimientos que se les pueden infligir a las víctimas, a los testigos y mayormente a los sujetos pasivos de un delito, incluso a través de las instituciones encargadas de hacer justicia. La terciaria son los estigmas que la sociedad le impone a la víctima (Novoa y Hernández, 2009).

Considero que la violación afectó no sólo a la familia pues, como he insistido, en los conflictos interétnicos la violación del cuerpo de la mujer representa la violación simbólica hacia el pueblo invadido. El cuerpo violado de la mujer representa la victoria del más fuerte sobre el vencido, logrando con esto el poder simbólico y político sobre los hombres indígenas (Segato, 2014; Yoc Cosajay, 2014).

Tomando en cuenta la perspectiva decolonial de Yoc Cosajay (2014) y de Segato (2014), la violación del cuerpo de la niña representó la violación de su comunidad y de su territorio. Así, la violación ocurrida en un contexto de lucha por las tierras, representa el poder de los mestizos sobre los indígenas. El cuerpo de la niña representa la victoria del mestizo, quien envía un mensaje a todos los que se atrevan a cuestionar el robo y el despojo de sus tierras, sembrando un ambiente de terror y presión psicosocial hacia la familia y otros miembros de la comunidad, quienes temen ser víctimas de nuevos ataques por parte de los agresores.

Comparto la visión de Segato $(2003,2016)$, quien afirma que la violación hacia las mujeres en contextos de violencia y luchas por el control del territorio no representa un acto que persigue un mero placer sexual, sino que el agresor viola el cuerpo principalmente para ejercer poder sobre su comunidad, para advertir y hacer valer su poder sobre los o'dami, orillándolos a abandonar sus tierras. 
18 Violación, cuerpo y cognición. Un caso en la Sierra Tarahumara

Ojeda Martínez, R. I.

\section{CONCLUSIONES ${ }^{2}$}

A lo largo del artículo se observó que la violación no sólo afectó a la víctima primaria, sino también a su familia y, en cierto sentido, a su grupo étnico asentado en su comunidad. Por lo tanto, la violación al cuerpo femenino en el contexto particular que se ha analizado, debe entenderse de forma integral, contemplando no sólo los daños a nivel de los individuos. Se vio cómo entre los o'dami las concepciones culturales de la persona están estrechamente ligadas a las concepciones sobre el cuerpo y el equilibrio entre el individuo, los otros y el universo (Rosas Mérida, 2006). Esto permitió entender el impacto social de la violación como un delito que resquebraja el tejido social de las comunidades indígenas.

Se puede afirmar que la violencia ejercida sobre los cuerpos es también practicada sobre las almas. Acorde con la visión del mundo o'dami, el sujeto violentado estaría en desequilibrio con el universo, con el cual forma una unidad, pues el cuerpo-soma. Es decir, el cuerpo, en tanto biología y materialidad, está en íntima correlación con el mundo físico y con el plano espiritual.

La concepción del cuerpo o'dami, como un complejo de interconexiones entre el cuerpo físico, espiritual y el mundo, resuena en el marco de las ideas de la cognición corporizada, lo cual puede ser sumamente útil para la antropología cognitiva, sobre todo para entender las diferencias entre los modelos cognitivos indígenas y los occidentales. Estas ideas también demuestran su utilidad en casos legales como éste, en el que fue necesario probar que en las culturas indígenas el daño a la víctima primaria implica también entender el daño en un sentido culturalmente situado.

Se analizó cómo la violación contra Esperanza no significa que el agresor haya sido motivado únicamente por un instinto sexual, sino que el motivo que está detrás de su acto estaba relacionado con una forma de dominación que los grupos criminales usan sobre los indígenas para tomar sus cuerpos, sus vidas y sus territorios (Segato, 2003, 2016).

El trabajo de investigación permitió entender cómo una familia o'dami -y por ende, la comunidad y el grupo étnico al que pertenecen- es desmembrada por la violencia que se vive en la Sierra Tarahumara, específicamente aquella ligada a las prácticas llevadas a cabo por los grupos criminales asociados al narcotráfico. Finalmente, es importante agregar que

\footnotetext{
${ }^{2}$ Agradezco profundamente a las personas o'dami que entrevisté en Guachochi y en la Ciudad de Chihuahua. También expreso mi agradecimiento a la asociación Alianza Sierra Madre por el acompañamiento durante la realización de peritaje que motivó esta investigación. Así mismo debo decir gracias a mi colega y amigo el dr. Juan Loera, por sus sugerencias y comentarios al primer borrador del texto. De manera especial agradezco al dr. Tom Froese por su valiosa asesoría y colaboración con relación al tema de la cognición corporizada. Finalmente, agradezco a las maestras Maritza Licón y Ana Murillo por su invaluable apoyo con las dudas de redacción, y a Karla Ivette Fierro Alvarado y Ana Karen Caballero por su ayuda en la transcripción de las entrevistas.
} 
es sustancial realizar más estudios sobre cómo se afectan las vidas de las niñas y adolescentes victimas de los delitos sexuales en la Sierra Tarahumara, pues las niñas son cuatro veces más vulnerables que el resto de la población, esto es a causa de su edad y de su género, y también por ser pobres e indígenas.

\section{REFERENCIAS}

Acosta, S. (2018). Cuerpo / territorio. Íconos-Revista de Ciencias Sociales, (61), 163-175. doi: https://doi.org/10.17141/iconos.61.2018.3316

Alarcón Gil, C. y Partido Lara, O. (2018). El caleidoscopio de las violencias en Chihuahua. En R. Zepeda Gil y S. Aguayo Quezada (Eds.), Construir el Estado, Construir la Paz: Memorias de la segunda conferencia sobre violencia y paz, (pp. 157-201). México: El Colegio de México/Instituto Belisario Domínguez.

Balbuena Mejía, Y. (2007). Producción y validación de conocimiento en el ciclo de vida entre los O'dami: Aproximaciones desde la racionalidad (Tesis de licenciatura). Escuela Nacional de Antropología e Historia, Ciudad de México.

Bonfil Sánchez, P., Marinis, N., Rosete Xotlanihua, B. y Martínez Navarro, R. (2017). Violencia de Género Contra Mujeres en Zonas Indígenas en México. México: Secretaría de Gobernación/Comisión Nacional para Prevenir y Erradicar la Violencia contra las Mujeres/Centro de Investigaciones y Estudios Superiores en Antropología Social/Consejo Nacional de Ciencia y Tecnología.

Breach Velducea, M. (19 de abril de 2007). Exigen indios tepehuanos posesión legal de tierras. La Jornada. Recuperado de https://www.jornada.com.mx/2007/04/19/index.php?section=estados\&article=039n1est

Breach Velducea, M. (6 de agosto de 2016). Destierra el narco a centenares de familias de la sierra de Chihuahua La Jornada. Recuperado de https://www.jornada.com.mx/2016/08/06/estados/022n1est

Chaparro, P. (2018). Violencia hacia la mujer en Baborigame, Guadalupe y Calvo, Chihuahua. (Tesis de maestría en Derechos Humanos). Universidad Autónoma de San Luis Potosí, México.

Company Fernández, A. (2014). Violencia expresiva e instrumental: La escena del crimen en homicidios cometidos en el seno de la pareja (Tesis de maestría en Criminología). Universidad de Barcelona, España.

Csordas, T. J. (1990). Embodiment as a Paradigm for Anthropology. Ethos, 18(1), 5-57. doi: https://doi.org/10.1525/eth.1990.18.1.02a00010

D'Andrade, R. G. (1995). The development of cognitive anthropology. Reino Unido: Cambridge University Press. 
20 Violación, cuerpo y cognición. Un caso en la Sierra Tarahumara

Ojeda Martínez, R. I.

De la Cruz, J. (2008). Culturas indígenas de la Nueva Vizcaya nuclear, siglos XVI y XVII [1er Coloquio Carl Lumholtz de Antropología e Historia del Norte de México]. En J. L. Sariego Rodríguez (Ed.), Retos de la Antropología en el Norte de México (pp. 115-169). Chihuahua: Escuela Nacional de Antropología e Historia.

De Marinis, N. (2013). Indigenous Rights and Violent State Construction: The strugle of Triqui Women of Oaxaca, Mexico. En R. Sieder y J. A. McNeish (Eds.), Gender Justice and Legal Pluralities:Latin American and African Perspectives, (pp. 156-179). Nueva York: Routledge Cavendish.

Denov, M. S. (2006). Wartime Sexual Violence: Assessing a Human Security Response to War-Affected Girls in Sierra Leone. Security Dialogue, 37(3), 319-342. doi: https://doi.org/10.1177/0967010606069178

Durt, C., Fuchs, T. y Tewes, C. (Eds.). (2017). Embodiment, Enaction, and Culture: Investigating the Constitution of the Shared World (1st. ed.). Londres: The MIT Press. Recuperado de https://mitpress.mit.edu/books/embodiment-enaction-and-culture

Frías, S. M. (2014). Ámbitos y formas de violencia contra mujeres y niñas: Evidencias a partir de las encuestas. Acta Sociológica, (65), 11-36. doi: https://doi.org/10.1016/S0186-6028(14)70235-X

Froese, T. (2019). Searching for the conditions of genuine intersubjectivity: From agent based models to perceptual corssing experiments. En A. Newen, L. De Bruin, y Gallagher (Eds.), The Oxford Handbook of 4E Cognition (pp. 163-186). Reino Unido: Oxford University Press.

Fuchs, T. (2018). Ecology of the brain: The phenomenology and biology of the embodied mind. Reino Unido: Oxford University Press.

Fulchirone, A., Paz, O. A., López, A. y Pérez, M. J. (2011). Tejidos que lleva el alma: Memoria de las mujeres mayas sobrevivientes de violación sexual durante el conflicto armado (2da. ed.). Guatemala: Equipo de Estudios Comunitarios y Acción Psicosocial/Unión Nacional de Mujeres Guatemaltecas.

Gonzalez-Grandón, X. y Froese, T. (2018). Grounding 4E Cognition in Mexico: Introduction to special issue on spotlight on 4E Cognition research in Mexico. Adaptive Behavior, 26(5), 189-198. doi: https://doi.org/10.1177/1059712318791633

Granham, A., Powell, M., Tylor, N., Anderson, D. y Fitzgerald, R. (2013). Investigación ética con niños. Florencia: Centro de Investigaciones de UNICEF/INNOCENTI.

Hernández Castillo, R. A. (2002). ¿Guerra Fraticida o estrategia etnocida? Las mujeres frente a la violencia política en Chiapas. En W. Jacorzynski (Ed.), Estudios sobre la violencia: teoría y práctica, (pp. 97-122). México: Centro de Investigaciones y Estudios Superiores en Antropología Social/Porrúa. 
Kirmayer, L. J. y Ramsted, M. J. D. (2017). Embodiment and Enactment in Cultural Psychiatry. En C. Durt, T. Fuchs y C. Tewes (Eds.), Embodiment, Enaction, and Culture: Investigating the Constitution of the Shared World, (pp. 397-422). Reino Unido: The MIT Press.

Lang, M. y Barrera Vivero, A. (Eds.). (2009). Mujeres indigenas y justicia ancestral. Quito: Fondo de Desarrollo de las Naciones Unidas para la Mujer.

Lindblom, J. y Ziemke, T. (2008). Interacting Socially through Embodied Action. In F. Morganti, A. Carassa, y G. Riva (Eds.), Enacting Intersubjectivity: A cognitive and social perspective to the study of interactions, (pp. 49-64). Ámsterdam: IOS Press.

Milán, M. (2017). El cuerpo como territorio. Bitácora Urbano Territorial, 27(3), 155-160. doi: https://doi.org/10.15446/bitacora.v27n3.66907

Misra, A. (2018). Towards a Philosophy of Narco Violence in Mexico. Londres: Palgrave Macmillan.

Montes Sánchez, A. y Salice, A. (2017). Feeling ashamed of myself because of you. In Ch. Durt, \& T. Fuchs (Eds.), Embodiment, Enaction, and Culture: Investigating the Constitution of the Shared World, (pp. 229-244). Cambrige MA: MIT Press.

Novoa Cota, V. J. y Hernández Sánchez, P. (2009). Efectos sociales y psicológicos de la violencia y la impunidad. En M. Gutiérrez Otero (Ed.), La violencia Sexual: Un problema internacional. Contextos socioculturales, (pp. 345-369). México: Universidad Autónoma de Ciudad Juárez.

Raíchali Noticias. (20 de octubre de 2019). Comunidades o'dami denuncian intento de despojo en la sierra de Chihuahua. La Verdad. Periodismo de investigación. Recuperado de https:/laverdadjuarez.com/index.php/2019/10/20/comunidades-odami-denuncianintento-de-despojo-en-la-sierra-de-chihuahua/

Ramos Lira, L. (2009). Abuso sexual y problemas de salud mental en adolescentes. En M. Gutiérrez (Ed.), La violencia sexual, un problema internacional, (pp. 79-103). México: Universidad Autónoma de Ciudad Juárez.

Reyes Valdez, J. A., Oseguera Montiel, A., Pacheco Bribiesca, R. C., y Saucedo Sánchez de Tagle, E. R. (2015). De la ambivalencia al tabú. Las transformaciones del concepto de persona en el Noroeste de México. En C. Good Eshelman y M. Alonso Bolaños (Coords.), Creando mundos, entrelazando realidades. Cosmovisiones y mitologías en el México indígena, (pp. 98-174). México: Consejo Nacional para la Cultura y las Artes/Instituto Nacional de Antropología e Historia.

Rosas Mérida, S. R. (2006). Concepción del cuerpo y la persona O'dami: La importancia del corazón como generador de pensamiento (Tesis de licenciatura). Escuela Nacional de Antropología e Historia, México. 
22 Violación, cuerpo y cognición. Un caso en la Sierra Tarahumara

Ojeda Martínez, R. I.

Rosas Mérida, S. R. (2012). Llegar y permanecer: O'dami los hilos que atan la vida. En A. Gutiérrez del Ángel (Ed.), Hilando al norte. Nudos, redes, textiles, (pp. 603-625). México: El Colegio de San Luis/El Colegio de la Frontera Norte.

Salgado Lévano, A. C. (2007). Investigación Cualitativa: Diseños, Evaluación del Rigor Metodoloógico y Retos. Liberabit, (13), 71-78.

Saucedo Sánchez de Tagle, E. R. (2004). Tepehuanes del Norte de México: Pueblos Indígenas del México Contemporáneo. México: Comisión Nacional para el Desarrollo de los Pueblos Indígenas. Recuperado de https://www.gob.mx/cms/uploads/attachment/file/12584/tepehuanes_norte.pdf

Segato, R. L. (2003). Las estructuras elementales de la violencia: Ensayos sobre género entre la antropología, el psicoanálisis y los derechos humanos. Buenos Aires: Universidad Nacional de Quilmes/Prometeo 3010.

Segato, R. L. (2014). Las nuevas formas de la guerra y el cuerpo de las mujeres. Sociedade e Estado, 29(2), 341-371. doi: https://doi.org/10.1590/S0102-69922014000200003

Segato, R. L. (2016). La guerra contra las mujeres. Madrid: Traficantes de sueños.

Sierra, M. T. (2008). Mujeres indígenas, justicia y derechos: Los retos de una justicia intercultural. Iconos Revista de Ciencias Sociales, (31), 15-26.

Varela, F. J., Thompson, E. y Rosch, E. (2011). De cuerpo presente: Las ciencias cognitivas y la experiencia humana. México: Gedisa Mexicana.

Vélez, J. C. (2009). Cognición hibrida, mente y representaciones externas. Revista de Educación y Pensamiento, (16), 47-62.

Videla Reyes, R., y Torrejón Vergara, A. (2019). ¿Tiempo objetivo de aprendizaje o temporalidad de la experiencia de aprendizaje en la escuela?: Aproximación ecológica de la cognición. En R. Videla (Coord). Pasos para una ecología cognitiva de la educación (pp. 15-35). Chile: Editorial Universidad de La Serena.

Villalobos, D., Martínez Esparza, P. y Carrillo Domínguez, H. (2018). Diagnóstico y propuestas sobre la violencia en la Sierra Tarahumara para la sociedad civil, comunidades, autoridades estatales y federales 2006-2017. Chihahua: CONTEC/FICOSEC.

Wood, E. J. (2009). Violencia sexual durante la guerra: Hacia un entendimiento de la variación. Análisis político, 22(66), 3-27.

Yoc Cosajay, A. M. (2014). Violencia sexual a mujeres indígenas durante el conflicto armado interno y el genocidio en Guatemala. Caravelle-Cahiers du monde hispanique et luso-brésilien, (102), 157-162. doi: https://doi.org/10.4000/caravelle.832 